\title{
The Radio Neutrino Observatory in Greenland (RNO-G)
}

\section{Stephanie Wissel ${ }^{a, *}$ on behalf of the RNO-G Collaboration}

(a complete list of authors can be found at the end of the proceedings)

${ }^{a}$ Pennsylvania State University, 104 Davey Lab, University Park, PA USA

E-mail: wissel@psu.edu

The Radio Neutrino Observatory Greenland (RNO-G) is scheduled for deployment in the summer of 2021. It will target the detection of astrophysical and cosmogenic neutrinos above $10 \mathrm{PeV}$. With 35 autonomous stations, it will be the largest implementation of a radio neutrino detector to date. The stations combine best-practice instrumentation from all previous radio neutrino arrays, such as a deep phased-array trigger and surface antennas. These proceedings describe the experimental considerations that have driven the design of RNO-G and the current progress in deployment, as well as discuss the projected sensitivity of the instrument. RNO-G will provide a unique view of the Northern Sky and will also inform the design of the radio component of IceCube-Gen2.

$37^{\text {th }}$ International Cosmic Ray Conference (ICRC 2021)

July 12th-23rd, 2021

Online - Berlin, Germany

\footnotetext{
*Presenter
} 


\section{Overview}

With the the discovery of a diffuse flux of astrophysical neutrinos [1,2] and the identification of the first candidate extra-galactic source of neutrinos [3, 4], neutrinos have been established a powerful messenger in the exploration of the high-energy universe. The Radio Neutrino Observatory in Greenland (RNO-G) will extend the reach of multi-messenger neutrino experiments to energies above $100 \mathrm{PeV}$ (ultra-high energies UHE, see Fig. 1), with a unique view of the Northern sky 3, and the largest footprint of a radio neutrino experiment (see Fig. 4), while also providing several critical technological tests that will inform the design of the radio component of IceCube-Gen2 [5, 6].

Neutrinos are excellent probes of high energy particle acceleration in the universe. Because they only interact through the weak force, neutrinos point back to their sources, can reach Earth from the most distant corners of the universe, and provide a clear indication of hadronic, cosmic ray acceleration. Above $100 \mathrm{PeV}$, astrophysical neutrinos can be generated through cosmic ray interactions with gas or radiation at (or near) some of the most energetic objects in the universe [7-13]. Cosmogenic neutrinos [14] expected due to interactions of cosmic rays with photon backgrounds $[15,16]-$ and from observations of the cosmic ray spectrum [17, 18] - are also expected at UHE [19-21]. The neutrino flux encodes information about the evolution and acceleration mechanisms at cosmological length scales.

The first observation of a neutrino from a blazar, TXS 0506+056, coincident with a flare in $\gamma$ rays opened a new window into the non-thermal universe through multi-messenger observations [ 3 , 4]. However, the neutrino sky must be more complex; neutrinos from blazars cannot comprise the bulk of the diffuse neutrino spectrum [22] and many sources may extend to higher energies [7-13]. Bursts of neutrinos from the most explosive sources are expected to peak in flux at the EeV scale. A full understanding of the neutrino sky over cosmological distances requires a large detector and observations expanded up to $\mathrm{EeV}$ energies.

RNO-G is an intermediate-scale discovery instrument for astrophysical and cosmogenic neutrinos at the highest energies that builds on prior ice radio experiments [23-28]. The design combines high single-station effective volume achieved with the deep array with a cosmic ray veto and enhanced the neutrino effective volume from a shallow array. Its scalable, low power, and autonomous design is a novel instrument design that provides important field testing for the larger radio array planned for IceCube-Gen2 [6]. In this proceeding, we describe the science prospects of RNO-G, the design, and the first deployment season.

\section{Science Prospects with a Large Radio Array in Greenland}

RNO-G's sensitivity to the diffuse flux of neutrinos in the UHE range is shown in the left panel of Fig. 1 as the $90 \%$ confidence level upper limit to the all-flavor diffuse flux of neutrinos. This is compared to several models of the expected cosmogenic flux of neutrinos, assuming a range of cosmic ray compositions that are still consistent with current observations from TA $[32,33]$ and Auger [19, 34]. These are representative samples of a large range of models [36, 37]. Within 5 years of observation, RNO-G is expected to constrain the diffuse flux.

Additionally, several models of astrophysical neutrinos are expected to contribute to the diffuse neutrino background in the UHE band. These models tend to extend to lower energies than the 


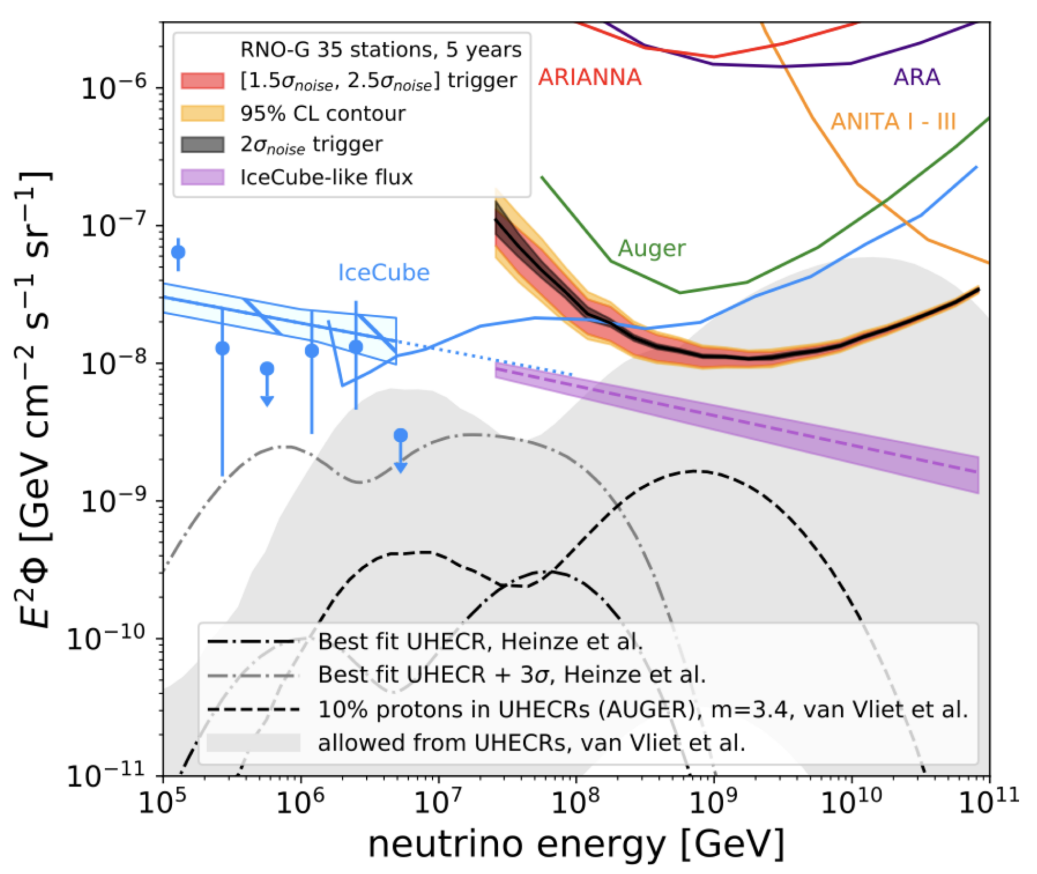

Figure 1: The five-year expected sensitivity of RNO-G to an all-flavor diffuse flux of neutrinos, assuming all five stations are active $66 \%$ of the time, compared with existing experiments [2, 29-31] and several representative cosmogenic fluxes [19, 32-34]. From Ref. [35].

cosmogenic models, but remain within the reach of RNO-G. Potential candidates range from Active Galactic Nuclei (AGN) [7] to various types of gamma-ray bursts (GRBs) [8, 9], pulsars [10], galaxy clusters [11], Flat Spectrum Radio Quasars (FSRQs) [12], and blazars [13].

RNO-G will also be capable of searching for transient phenomena that generate UHE neutrinos, extending multi-messenger observations of these explosive objects into a new energy range. RNOG's neutrino fluence sensitivity in a range of zenith angle bands is shown in the right panel of Fig. 2. Flares of blazars $[12,38]$ may generate UHE neutrinos along with neutrinos at lower energies observable with IceCube. Gamma-ray bursts (GRBs) can produce UHE neutrinos at several stages in their evolution, including during the prompt phase of short GRBs [39] and in the afterglow phase [9]. Cataclysmic events like tidally disrupted stars [40] and neutron star mergers [41, 42], some of which can result in long-lived magnetars [43], can produce gravitational waves and neutrinos simultaneously. A planned design feature of RNO-G is the capability to send and respond to real-time alerts, made possible by a local LTE network and continuous satellite network connection between Summit Station and RNO-G institutions.

RNO-G will provide a unique view of the UHE Northern sky that is broad in declination and overlaps with the region of the sky where IceCube is most sensitive to lower-energy neutrinos, as shown in Fig. 3. The Earth is opaque to neutrinos at PeV to EeV energies, such that UHE neutrino observatories are most sensitive to Earth-skimming neutrinos. Therefore, follow-up of TeV-scale IceCube events at higher energies requires a Northern detector. In addition to the targets defined by IceCube, other sources visible with RNO-G include several well-known TeV gamma ray blazars ${ }^{1}$

${ }^{1}$ http://tevcat.uchicago.edu 


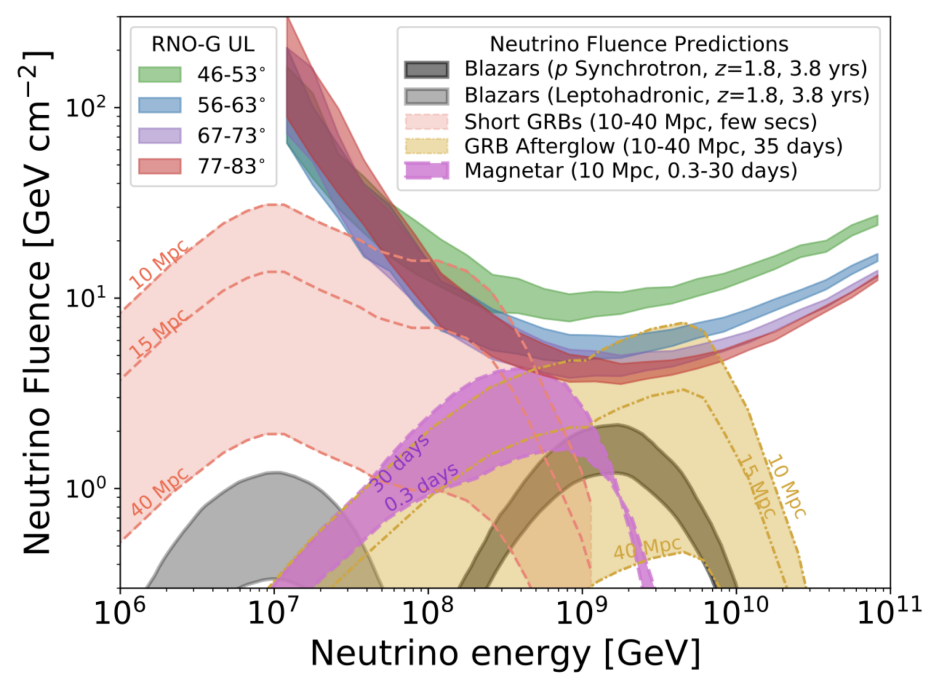

Figure 2: The neutrino fluence sensitivity (95\% CL, full decade in energy) of RNO-G shown in several zenith angle bands. Several models for transient sources including blazars [38], stable magnetars [43], short GRBs [39], GRB afterglows [9] are shown for comparison at several luminosity distances. From Ref. [35].

and the TA hospot [44]. The broad sky coverage will enables studies of studies of point sources of high-energy neutrinos in the UHE band that can be combined with neutrino observations at lower energies.

RNO-G will also provide a testbed for fundamental physics at energies in a new energy range (see Ref. [45, 46] and references therein). Nucleon and nuclear structure and potential new physics can be probed through cross-section measurements of the interactions of neutrinos with nucleons. Some models, such as those that include sphaelerons, supersymmetry, rolled-up dimensions, or leptoquarks, can lead to drastic increases in the cross-section at UHE energies. They can also be used to measure probe parton distributions at low Bjorken $x$ and high $Q^{2}$, well beyond the reach of the Large Hadron Collider. Fundamental properties of the neutrino can be explored at a new energy scale and over long length scales including flavor oscillations and neutrino decay. Several opportunities are available for exploring new physics including dark matter, neutrino selfinteractions, fundamental symmetries, and interactions of neutrinos with dark backgrounds, among others. All fundamental physics measurements require the discovery of UHE neutrinos and good energy and angular resolution.

\section{The Radio Technique in Greenland}

The radio detection technique relies on coherent, impulsive radio signals emitted when a neutrino interacts in a dense material [48], due to a negative charge excess in the resulting cascade. For wavelengths larger than the transverse size of the shower, the emission is coherent, leading to a large power boost for high-energy showers at frequencies $\lesssim 1 \mathrm{GHz}$. This so-called Askaryan effect [49] has been demonstrated in beam-test experiments [50-53]. The radio technique has advanced over the last two decades through several experiments [27, 54], especially recently through 


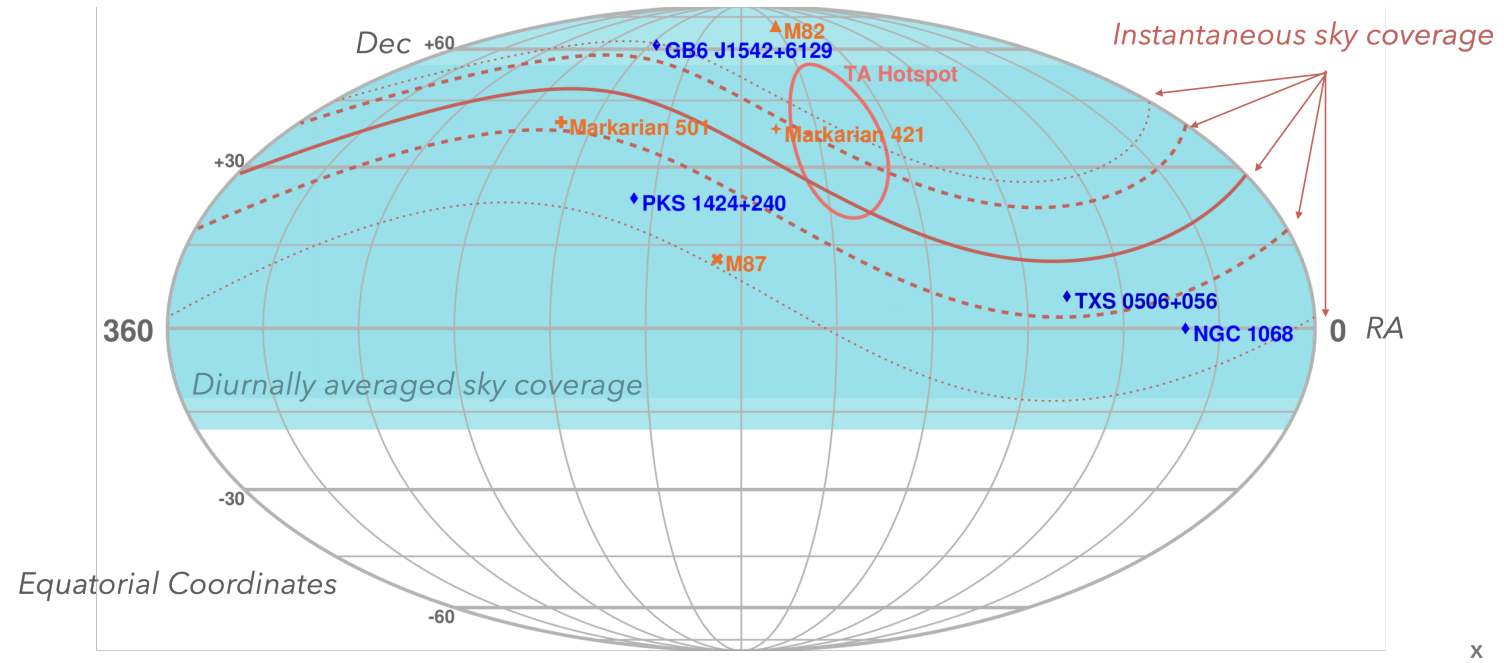

Figure 3: Sky coverage of RNO-G compared with several potential sources indicating the unique view of the Northern sky accessible to RNO-G, shown as both the instantaneous sky coverage and the diurnally averaged sky coverage. Shown in blue are the most significant sources in a point-source search in IceCube [47]. The orange sources include strong gamma ray or radio emitters as well as the TA hotspot [44]. From Ref. [35].

the balloon-borne experiment ANITA [27], the ARIANNA shallow array [55] and ARA, a deep-ice detector at the South Pole $[23,56]$. These and other radio instruments have further validated the radio technique through cosmic ray observations [57-59] and radio propagation studies [60, 61].

RNO-G is located at Summit Station, a site managed by the US National Science Foundation (NSF). Summit Station $\left(72^{\circ} 35^{\prime} 46^{\prime \prime}\right.$ N, $\left.38^{\circ} 25^{\prime} 19^{\prime \prime} \mathrm{W}\right)$ is located in near the apex of the ice sheet in Greenland over $3.2 \mathrm{~km}$ of ice. Early field campaigns established that the site is suitable for a large scale neutrino detector. In 2013, the depth averaged attenuation length was first measured at Summit Station [62] to be $823_{-209}^{+189} \mathrm{~m}$ when extrapolated from $75 \mathrm{MHz}$ to $300 \mathrm{MHz}$. In 2015, the field teams deployed an analog prototype of the trigger design used in RNO-G. They also monitored both the radio frequency noise environment [62] and radio wave propagation in the upper layer of snow known as the firn [63]. Further ice studies relevant for radio neutrino detection are underway at Summit Station now.

\section{Instrument Design}

As shown in Fig. 4, RNO-G will be a large scale observatory consisting of 35 independentlytriggered stations separated by $1.25 \mathrm{~km}$. The array will cover a $50 \mathrm{~km}^{2}$ area over the surface of glacial ice. While the first stations were already deployed in the summer of 2021, the array is planned to be completed and fully operational by 2024 .

The RNO-G design combines a deep array for high-sensitivity triggering and a surface array for a cosmic-ray veto and improved event reconstruction. Most of the deep antennas, both vertically polarized (Vpol) and horizontally polarized (Hpol) antennas, are deployed on a single string of instrumentation in one hole. The other two instrumented strings provide azimuthal reconstruction and also have impulsive calibration sources for in situ characterization. The hardware for RNO-G is described in detail in these proceedings [64]. 


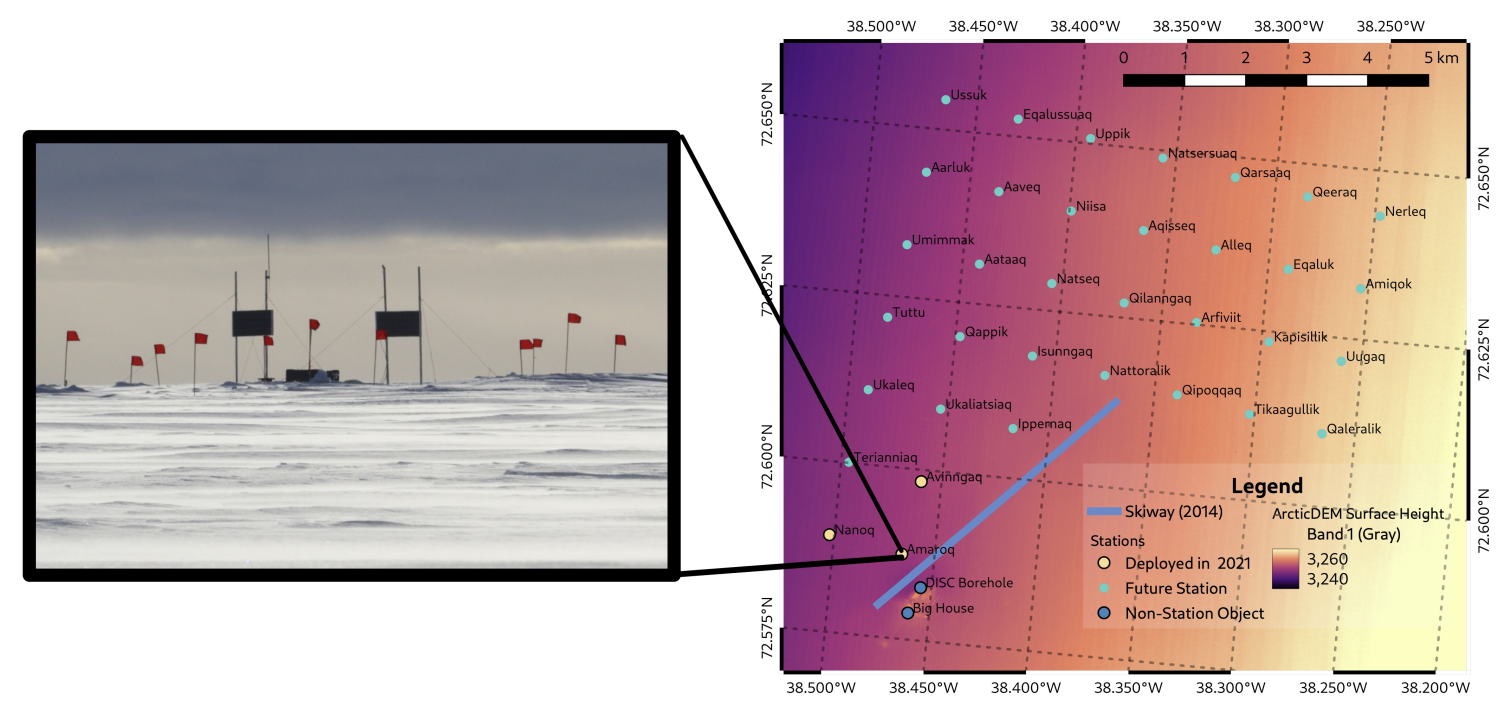

Figure 4: Planned RNO-G array at Summit Station, including the first 3 stations deployed in 2021. The first station deployed, Amaroq, is shown on the left.

The RNO-G stations, shown in Fig. 5, each have 15 deep channels and 9 shallow channels. The power string, shown on the left, has a compact, closely packed array of antennas used in the primary trigger as well as antennas deployed at different depths along the same string. The helper strings provide additional antennas for reconstruction and calibration pulsers. The vertically polarized antennas consist of 5-inch diameter fat dipoles based on the RICE and GNO dipole designs. The horizontally polarized antennas use an 8-inch diameter aluminum quad-slot design. Commercially available LPDAs are used for the for the shallow channels. Three of the LPDAs point upward, while the remaining six point downward.

Because the density and index of refraction of ice increases with depth, radio signals from neutrino interactions deep in the ice bend as they travel towards the surface. Antennas positioned deeper in the ice therefore access a larger volume of ice and fractional field of view than those at the surface, which motivates using the deep channels as the primary trigger.

RNO-G stations are primarily triggered with an interferometric phased array on the deep vertically polarized channels, based on a similar trigger used in the ARA experiment [65] and tested in early field campaigns in Greenland [62]. The phased array trigger coherently sums the envelopes of the waveforms with time delays corresponding to a range of angles of incident plane waves, increasing the voltage signal-to-noise ratio (SNR) for triggering by the square root of the number of antennas in the array [65]. Based on a full Askaryan trigger simulation, validated by the performance of the existing ARA system, we expect to achieve an elevation-averaged $50 \%$ trigger efficiency point at a $2 \sigma$ threshold in voltage. The trigger for RNO-G is tuned to the lower portion of the frequency band (80-236 MHz), which optimizes for events further from the Cherenkov cone and achieves a comparable trigger efficiency as the ARA phased array even with fewer channels [35, 66]. Each station uses a custom designed digitizer board using LAB4d digitizers [67], which yield more than a GHz of bandwidth, using two buffers of 2048 samples each.

Cosmic ray air showers provide a unique opportunity for instrument calibration and validation. 


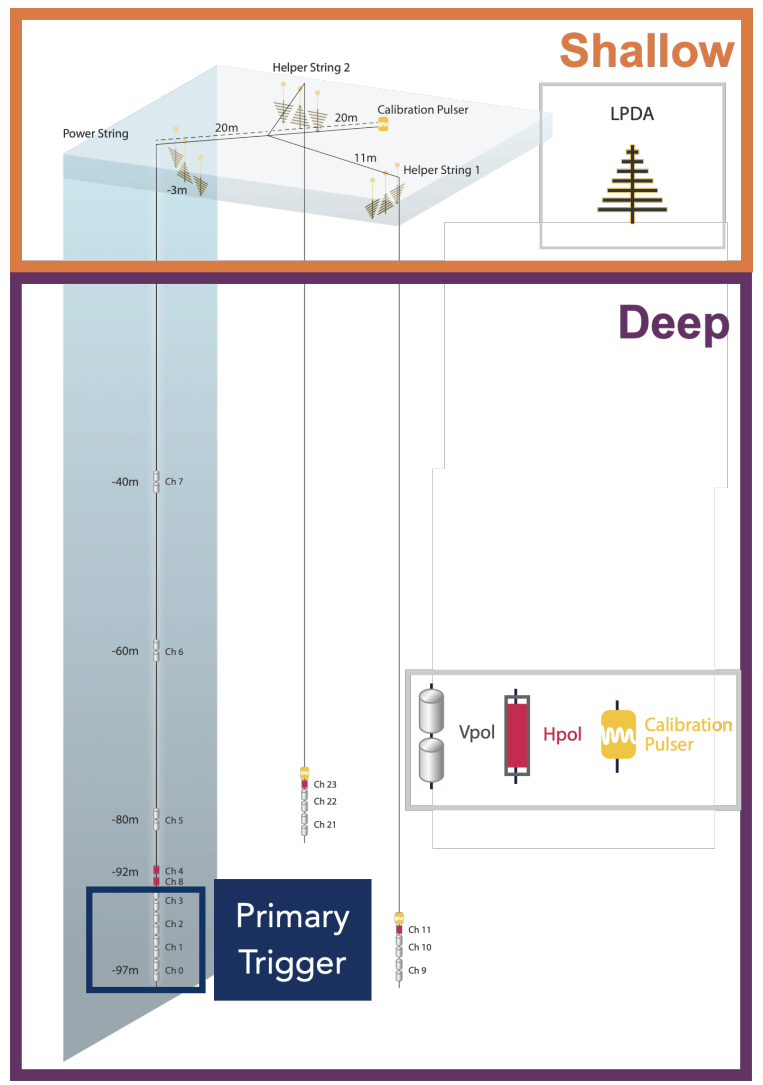

Figure 5: Isometric view of an RNO-G station. The as-deployed design shown here incorporates shallow and deep antennas to search for ultra-high energy neutrinos.

Air showers from downgoing cosmic rays generate coherent radio emission from the combined effects of geomagnetic emission and Askaryan radiation, with geomagnetic emission dominating. Geomagnetic emission occurs due to the currents induced by the Lorentz force acting on charged particles in the shower. The expected radio footprint for a range of zenith angles is shown in Fig. 6. Cosmic ray and neutrino radio signals can be distinguished by their polarization, signal shape $[57,68,69]$, but because both signals are broadband and impulsive, the more prevalent cosmic ray events can be used to calibrate the instrument timing and energy scale [58, 59]. Using an independent trigger with only the shallow channels, we expect $O(10-100)$ cosmic ray events per day. Because the radio signal refracts into a narrow range of angles near the nulls of the deep antennas, we expect the cosmic ray rate using the primary trigger formed with the deep channels to be substantially lower. However, the instrument sensitivity can be calibrated with the surface channels.

The same characteristics that make cosmic rays a good calibration source also make them potential backgrounds. Thus the shallow channels provide an additional veto for cosmic ray events. This is especially important for more insidious cosmic-ray induced backgrounds like cosmic-ray muons that generate showers in the ice similar to neutrinos [70] and transition radiation from air showers impacting the ice [71]. The shallow channels can also veto non-thermal backgrounds originating from above the surface. These potential cosmic ray backgrounds can be $O(0.1-1)$ events 

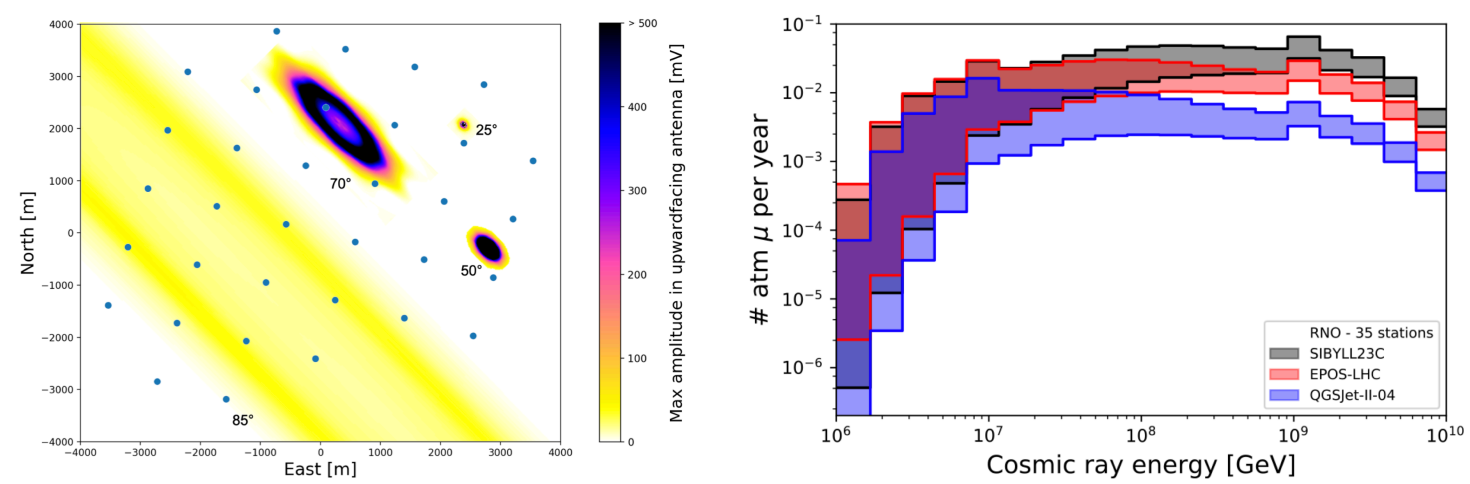

Figure 6: (Left) The radio footprint of $3.2 \times 10^{18} \mathrm{eV}$ cosmic ray air showers over the full RNO-G array, simulated with CORSIKA. (Right) The number of detected atmospheric muons per year as a function of shower energy in a simulated array as a function of cosmic ray energy. From Ref. [35, 70].
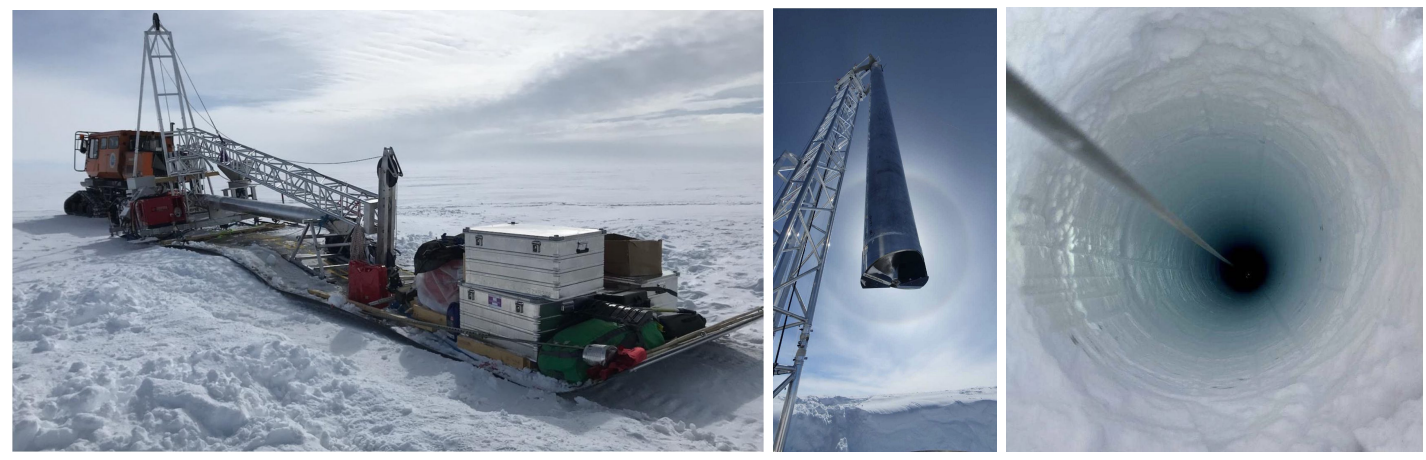

Figure 7: The Big RAID Auger drill designed by the British Antarctic Survey to drill 11-inch diameter holes.

per array per year, which may be comparable to rate of neutrinos.

The stations are autonomous, using solar power to provide an expected 60-70\% uptime and an LTE cellular network for the high-throughput communications required to transmit the full waveforms back to the Big House. A backup lower throughput LoRaWAN that can be used for station commanding and monitoring is also available. Summit Station has a continuous, 24-hour satellite connection that allows for $1 \mathrm{~Gb}$ /day of data to be transmitted to the RNO-G collaborating institutions. The continuous networking capabilites can be used to respond to and generate multimessenger alerts.

\section{The First Deployment Season}

The first three stations, Amaroq (shown in the left panel of Fig. 4), Nanoq, and Avinngaq, were deployed at Summit Station during this conference, in the summer of 2021. These stations are the closest to the main Summit Station. They are also the closest planned stations to a deep hole, the DISC (Deep Ice-Sheet Coring) borehole [72], an full-depth borehole available for use in calibrating 


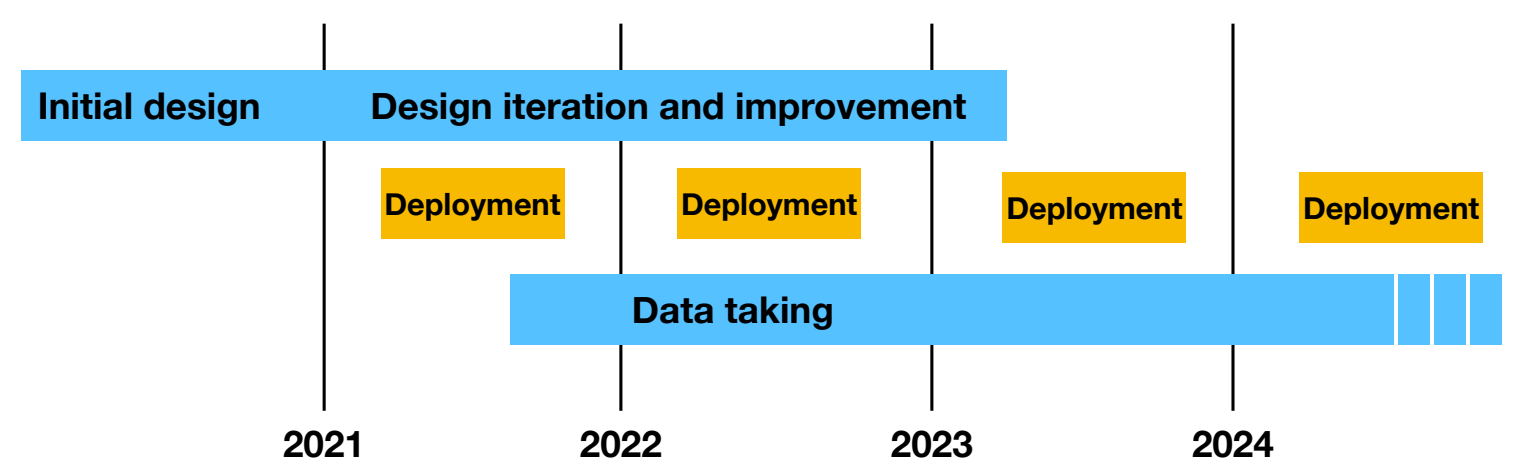

Figure 8: Deployment schedule for RNO-G.

the stations over long distances. The remaining stations are expected to be deployed in by 2024 . The deployment schedule shown in Fig. 8 includes delays due to the COVID-19 pandemic.

Three 100-m holes are drilled per station using the custom designed 11-inch drill (the British Antarctic Survey's BigRAID drill [73]), shown in Fig. 7. Over the 2021 deployment season most holes were drilled in two shifts of two people per hole, but we anticipate improvements to the drilling procedure that can complete a 100-m hole in one shift. Aspects of the hardware and station deployment procedure are illustrated in Fig. 9.

Fig. 10 shows some of the first events recorded on the vertically polarized channels in Amaroq. These events were generated by a field team member driving a snowmobile near the station. The events demonstrate the impulsive character of the signals searched for by RNO-G.

\section{Conclusions}

RNO-G is a mid-scale UHE neutrino detector currently under construction and operating at Summit Station in Greenland. It is the first science-level instrument to target the UHE cosmic neutrino sky in the North. The novel hybrid design combines deep component for high effective volume from deep, low threshold trigger with shallow component for improved angular reconstruction, additional neutrino sensitivity, and background vetos. The instrument is designed to be scalable, both allowing a large number of stations to be installed in over four years and using a low power design.

We are thankful to the staff at Summit Station for supporting our deployment work in every way possible. Also to our colleagues from the British Antarctic Service for getting excited about building and operating the BigRAID drill for our project. We would like to acknowledge our home institutions and funding agencies for supporting the RNO-G work; in particular the Belgian Funds for Scientific Research (FRS-FNRS and FWO) and the FWO programme for International Research Infrastructure (IRI), the National Science Foundation through the NSF Awards 2118315 and 2112352 and the IceCube EPSCoR Initiative (Award ID 2019597), the German research foundation (DFG, Grant NE 2031/2-1), the Helmholtz Association (Initiative and Networking Fund, W2/W3 Program), the University of Chicago Research Computing Center, and the European Research Council under the European Unions Horizon 2020 research and innovation programme (grant agreement No 805486). 

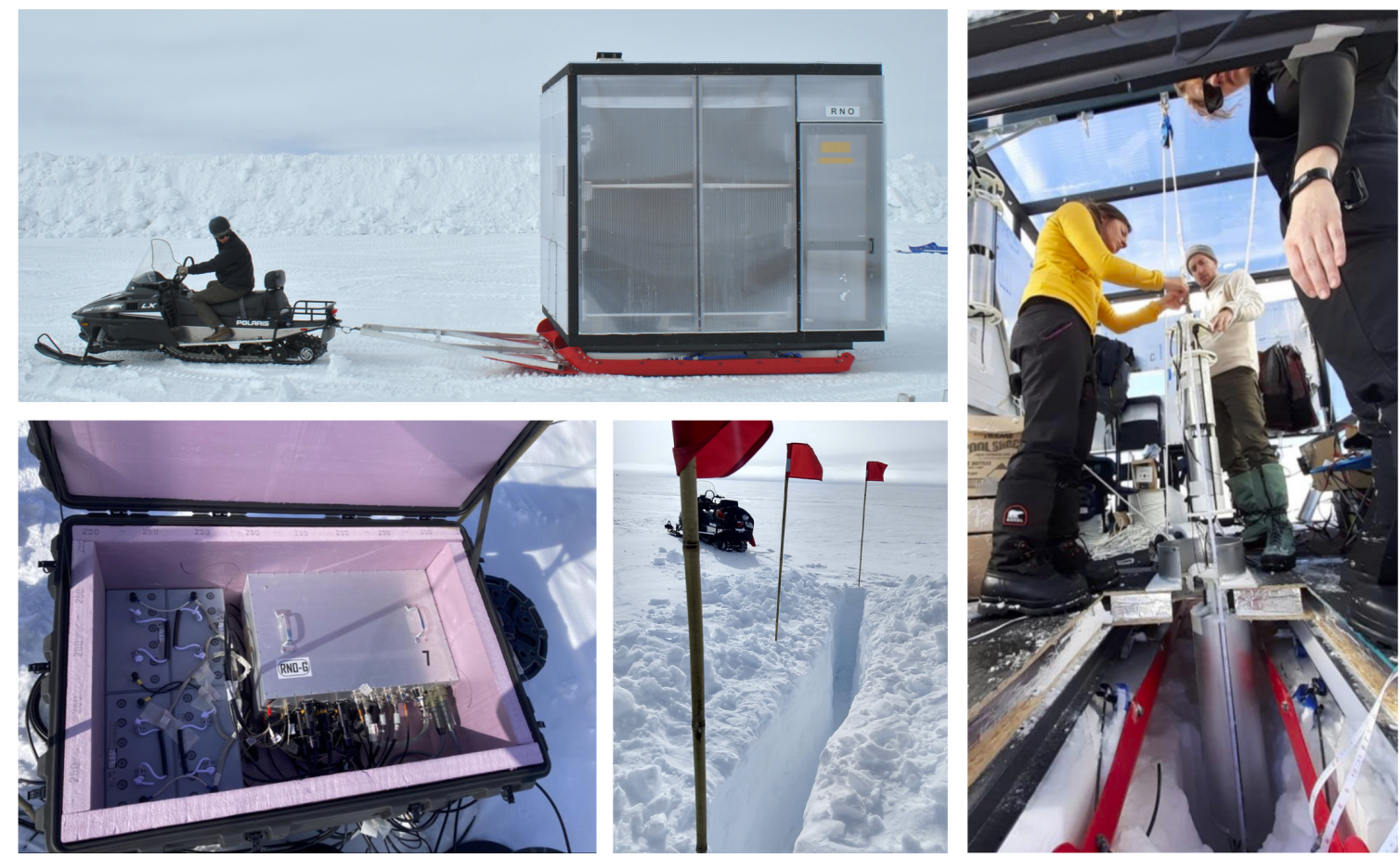

Figure 9: Deployment of the RNO-G stations proceeds after the three holes are drilled. The snow-mobile driven deployment hut (top left) is secured over the holes, so that the deep component of the station can be installed at their final depth (right). Shallow antennas (trench shown in bottom middle), cables, solar panels, and the vault (bottom left) containing the data acquisition system, batteries and interfaces to the antennas are installed directly at the surface above the array.
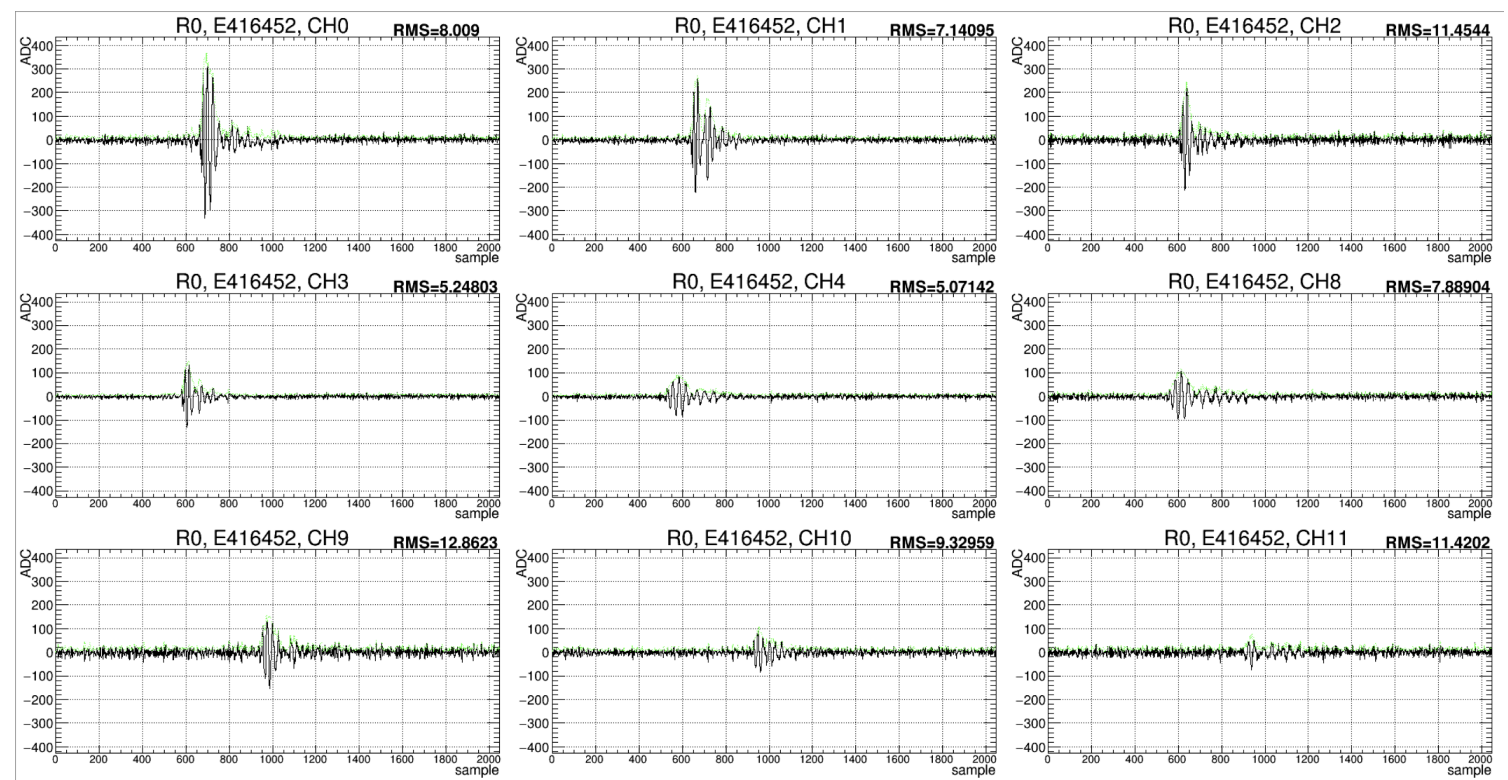

Figure 10: Impulsive events from snowmobiles recorded on the vertically polarized channels of the deep component in the first station, Amaroq. 


\section{References}

[1] M. G. Aartsen, et al., Evidence for High-Energy Extraterrestrial Neutrinos at the IceCube Detector, Science 342 (2013) 1242856. arXiv: 1311.5238, doi : 10.1126/science. 1242856.

[2] M. G. Aartsen, et al., A Measurement of the Diffuse Astrophysical Muon Neutrino Flux Using Eight Years of IceCube Data, PoS ICRC2017 (2017) 1005. arXiv: 1710.01191.

[3] M. G. Aartsen, et al., Multimessenger observations of a flaring blazar coincident with highenergy neutrino IceCube-170922A, Science 361 (6398) (2018) eaat1378. arXiv: 1807. 08816, doi:10.1126/science. aat1378.

[4] M. G. Aartsen, et al., Neutrino emission from the direction of the blazar TXS 0506+056 prior to the IceCube-170922A alert, Science 361 (6398) (2018) 147-151. arXiv: 1807.08794, doi:10.1126/science . aat2890.

[5] M. Aartsen, et al., IceCube-Gen2: The Window to the Extreme Universe (8 2020). arXiv: 2008.04323.

[6] S. Hallman, B. Clark, C. Glaser, D. Smith, et al., Sensitivity studies for the IceCube-Gen2 radio array, PoS ICRC2021 (2021) 1183. doi : 10.22323/1.395.1183.

[7] K. Murase, Y. Inoue, C. D. Dermer, Diffuse neutrino intensity from the inner jets of active galactic nuclei: Impacts of external photon fields and the blazar sequence, Phy. Rev. D90 (2014) 023007.

[8] D. Boncioli, D. Biehl, W. Winter, On the common origin of cosmic rays across the ankle and diffuse neutrinos at the highest energies from low-luminosity Gamma-Ray Bursts, Astrophys. J. 872 (2019) 110. arXiv: 1808.07481, doi : 10.3847/1538-4357/aafda7.

[9] K. Murase, High energy neutrino early afterglows gamma-ray bursts revisited, Phys. Rev. D76 (2007) 123001. arXiv:0707.1140, doi:10.1103/PhysRevD .76.123001.

[10] K. Fang, et al., Testing the Newborn Pulsar Origin of Ultrahigh Energy Cosmic Rays with EeV Neutrinos, Phys. Rev. D90 (10) (2014) 103005. arXiv:1311.2044, doi:10.1103/ PhysRevD.90.103005, 10.1103/PhysRevD.92.129901.

[11] K. Fang, K. Murase, Linking High-Energy Cosmic Particles by Black Hole Jets Embedded in Large-Scale Structures, Phys. Lett. 14 (2018) 396, nature Phys.14,no.4,396 (2018). arXiv: 1704.00015, doi : 10.1038/s41567-017-0025-4.

[12] C. Righi, A. Palladino, F. Tavecchio, F. Vissani, EeV Astrophysical neutrinos from FSRQs? (2020). arXiv:2003.08701.

[13] X. Rodrigues, J. Heinze, A. Palladino, A. van Vliet, W. Winter, Blazar origin of the UHECRs and perspectives for the detection of astrophysical source neutrinos at EeV energies (2020). arXiv:2003.08392. 
[14] V. Berezinsky, G. Zatsepin, Cosmic rays at ultrahigh-energies (neutrino?), Phys. Lett. B 28 (1969) 423. doi : 10 .1016/0370-2693(69)90341-4.

[15] K. Greisen, End to the cosmic ray spectrum?, Phys. Rev. Lett. 16 (1966) 748-750. doi: 10.1103/PhysRevLett. 16.748.

[16] G. T. Zatsepin, V. A. Kuzmin, Upper limit of the spectrum of cosmic rays, JETP Lett. 4 (1966) 78-80.

[17] A. Castellina, Highlights from the Pierre Auger Observatory (ICRC2019), PoS ICRC2019 (2020) 004. arXiv: 1909.10791, doi:10.22323/1.358.0004.

[18] S. Ogio, Highlights from the Telescope Array experiment (ICRC2019), PoS ICRC2019 (2020) 013. doi: $10.22323 / 1.358 .0234$.

[19] J. Heinze, et al., A new view on Auger data and cosmogenic neutrinos in light of different nuclear disintegration and air-shower models, Astrophys. J. 873 (2019) 88. arXiv: 1901. 03338, doi: $10.3847 / 1538-4357 /$ ab05ce.

[20] A. Romero-Wolf, M. Ave, Bayesian Inference Constraints on Astrophysical Production of Ultra-high Energy Cosmic Rays and Cosmogenic Neutrino Flux Predictions, JCAP 1807 (2018) 025. arXiv: 1712.07290, doi: 10.1088/1475-7516/2018/07/025.

[21] R. Alves Batista, R. M. de Almeida, B. Lago, K. Kotera, Cosmogenic photon and neutrino fluxes in the Auger era, JCAP 1901 (2019) 002. arXiv: 1806.10879, doi:10.1088/ $1475-7516 / 2019 / 01 / 002$.

[22] M. G. Aartsen, et al., The contribution of Fermi-2LAC blazars to the diffuse TeV-PeV neutrino flux, Astrophys. J. 835 (2017) 45. arXiv: 1611. 03874, doi : 10 . 3847/1538-4357/835/1/ 45.

[23] P. Allison, et al., Design and Initial Performance of the Askaryan Radio Array Prototype EeV Neutrino Detector at the South Pole, Astropart. Phys. 35 (2012) 457-477. arXiv: 1105 . 2854, doi:10.1016/j. astropartphys.2011.11.010.

[24] P. Allison, et al., Constraints on the Diffuse Flux of Ultra-High Energy Neutrinos from Four Years of Askaryan Radio Array Data in Two Stations, Phys. Rev. D 102 (2020) 043021. arXiv: 1912.00987, doi:10.1103/PhysRevD.102.043021.

[25] S. W. Barwick, et al., A First Search for Cosmogenic Neutrinos with the ARIANNA Hexagonal Radio Array, Astropart. Phys. 70 (2015) 12-26. arXiv:1410.7352, doi:10.1016/j. astropartphys.2015.04.002.

[26] A. Anker, et al., A search for cosmogenic neutrinos with the ARIANNA test bed using 4.5 years of data, JCAP 03 (2020) 053. arXiv: 1909.00840, doi : 10 . 1088/1475-7516/2020/ 03/053. 
[27] P. W. Gorham, et al., The Antarctic Impulsive Transient Antenna Ultra-high Energy Neutrino Detector Design, Performance, and Sensitivity for 2006-2007 Balloon Flight, Astropart. Phys. 32 (2009) 10-41. arXiv:0812.1920, doi:10.1016/j . astropartphys. 2009.05.003.

[28] P. Gorham, et al., Constraints on the ultrahigh-energy cosmic neutrino flux from the fourth flight of ANITA, Phys. Rev. D 99 (12) (2019) 122001. arXiv: 1902 .04005, doi:10.1103/ PhysRevD.99.122001.

[29] A. Aab, et al., Improved limit to the diffuse flux of ultrahigh energy neutrinos from the Pierre Auger Observatory, Phys. Rev. D 91 (2015) 092008. arXiv: 1504.05397, doi: 10.1103/PhysRevD. 91.092008.

[30] P. W. Gorham, et al., Constraints on the diffuse high-energy neutrino flux from the third flight of ANITA, Phys. Rev. D98 (2) (2018) 022001. arXiv: 1803.02719, doi:10.1103/ PhysRevD.98.022001.

[31] P. Allison, et al., Performance of two Askaryan Radio Array stations and first results in the search for ultrahigh energy neutrinos, Phys. Rev. D93 (8) (2016) 082003. arXiv: 1507 . 08991 , doi:10.1103/PhysRevD.93.082003.

[32] A. van Vliet, CRPropa simulations, similar to PoS(ICRC2019)190, private communication (2019).

[33] D. Bergmann for the Telescope Array Collaboration, Combined fit of the spectrum and composition from telescope array, PoS(ICRC2019) (2019) 190.

[34] A. Aab, et al., Combined fit of spectrum and composition data as measured by the Pierre Auger Observatory, JCAP 2017 (2017) 038. doi : 10 . 1088/1475-7516/2017/04/038.

[35] J. A. Aguilar, et al., Design and Sensitivity of the Radio Neutrino Observatory in Greenland (RNO-G), JINST 16 (03) (2021) P03025. arXiv: 2010 . 12279, doi : 10 . 1088/1748-0221/ 16/03/P03025.

[36] A. van Vliet, R. Alves Batista, J. R. Hörandel, Determining the fraction of cosmic-ray protons at ultrahigh energies with cosmogenic neutrinos, Phys. Rev. D100 (2) (2019) 021302. arXiv: 1901.01899, doi:10.1103/PhysRevD.100.021302.

[37] M. S. Muzio, G. R. Farrar, M. Unger, Ultrahigh energy cosmic rays and high energy astrophysical neutrinos (8 2021). arXiv:2108.05512.

[38] X. Rodrigues, et al., Multi-wavelength and neutrino emission from blazar pks $1502+106$, arXiv preprint (2020). arXiv:2009.04026.

[39] S. S. Kimura, . Murase, P. Mészáros, K. Kiuchi, High-Energy Neutrino Emission from Short Gamma-Ray Bursts: Prospects for Coincident Detection with Gravitational Waves, Astrophys. J. 848 (2017) L4. arXiv: 1708 .07075, doi : 10 . 3847/2041-8213/aa8d14. 
[40] C. Guépin, et al., Ultra-High Energy Cosmic Rays and Neutrinos from Tidal Disruptions by Massive Black Holes, Astron. Astrophys. 616 (2018) A179. arXiv: 1711.11274, doi: $10.1051 / 0004-6361 / 201732392$.

[41] K. Fang, B. D. Metzger, High-Energy Neutrinos from Millisecond Magnetars formed from the Merger of Binary Neutron Stars, Astrophys. J. 849 (2) (2017) 153. arXiv: 1707.04263 , doi : $10.3847 / 1538-4357 /$ aa8b6a.

[42] S. S. Kimura, K. Murase, I. Bartos, K. Ioka, I. S. Heng, P. Mészáros, Transejecta highenergy neutrino emission from binary neutron star mergers, Phys. Rev. D98 (2018) 043020. doi : 10.1103/PhysRevD. 98.043020.

[43] K. Fang, B. D. Metzger, High-Energy Neutrinos from Millisecond Magnetars formed from the Merger of Binary Neutron Stars, Astrophys. J. 849 (2017) 153. arXiv: 1707.04263, doi : $10.3847 / 1538-4357 /$ aa8b6a.

[44] R. U. Abbasi, et al., Indications of Intermediate-Scale Anisotropy of Cosmic Rays with Energy Greater Than $57 \mathrm{EeV}$ in the Northern Sky Measured with the Surface Detector of the Telescope Array Experiment, Astrophys. J. 790 (2014) L21. arXiv: 1404.5890, doi: $10.1088 / 2041-8205 / 790 / 2 / \mathrm{L} 21$.

[45] M. Ackermann, et al., Fundamental physics with high-energy cosmic neutrinos, Bull. Am. Astron. Soc. 51 (3) (3) (2019) 215.

[46] S. R. Klein, Probing high-energy interactions of atmospheric and astrophysical neutrinos, World Scientific. ISBN: 978-981-3275-01-0, 2020, Ch. 4, pp. 75-107. arXiv: 1906.02221, doi : 10.1142/9789813275027\_0004.

[47] M. Aartsen, et al., Time-Integrated Neutrino Source Searches with 10 Years of IceCube Data, Phys. Rev. Lett. 124 (5) (2020) 051103. arXiv: 1910 .08488, doi : 10 .1103/PhysRevLett. 124.051103.

[48] A. Connolly, A. G. Vieregg, Radio Detection of High Energy Neutrinos, Neutrino Astronomy - Current status, future prospects, Eds. T. Gaisser \& A. Karle (World Scientific) (2017) 217-240arXiv: 1607.08232, doi:10.1142/9789814759410_0015.

[49] G. A. Askar'yan, Excess negative charge of an electron-photon shower and its coherent radio emission, Sov. Phys. JETP 14 (2) (1962) 441-443.

[50] D. Saltzberg, et al., Observation of the Askaryan effect: Coherent microwave Cherenkov emission from charge asymmetry in high-energy particle cascades, Phys. Rev. Lett. 86 (2001) 2802-2805. arXiv:hep-ex/0011001, doi:10.1103/PhysRevLett.86.2802.

[51] P. W. Gorham, et al., Observations of the Askaryan effect in ice, Phys. Rev. Lett. 99 (2007) 171101. arXiv:hep-ex/0611008, doi:10.1103/PhysRevLett.99.171101.

[52] P. W. Gorham, et al., Accelerator measurements of the Askaryan effect in rock salt: A Roadmap toward teraton underground neutrino detectors, Phys. Rev. D72 (2005) 023002. arXiv: astro-ph/0412128, doi:10.1103/PhysRevD.72.023002. 
[53] K. Belov, et al., Accelerator measurements of magnetically-induced radio emission from particle cascades with applications to cosmic-ray air showers, Phys. Rev. Lett. 116 (14) (2016) 141103. arXiv: 1507.07296, doi:10.1103/PhysRevLett.116.141103.

[54] I. Kravchenko, et al., Rice limits on the diffuse ultrahigh energy neutrino flux, Phys. Rev. D73 (2006) 082002. arXiv:astro-ph/0601148, doi:10.1103/PhysRevD . 73.082002.

[55] L. Gerhardt, S. Klein, T. Stezelberger, S. Barwick, K. Dookayka, J. Hanson, R. Nichol, A prototype station for ARIANNA: a detector for cosmic neutrinos, Nucl. Instrum. Meth. A624 (2010) 85-91. arXiv: 1005.5193, doi:10.1016/j .nima.2010.09.032.

[56] P. Allison, et al., First Constraints on the Ultra-High Energy Neutrino Flux from a Prototype Station of the Askaryan Radio Array, Astropart. Phys. 70 (2015) 62-80. arXiv: 1404 . 5285, doi: 10.1016/j . astropartphys.2015.04.006.

[57] S. Hoover, et al., Observation of Ultra-high-energy Cosmic Rays with the ANITA Balloonborne Radio Interferometer, Phys. Rev. Lett. 105 (2010) 151101. arXiv: 1005.0035, doi: 10.1103/PhysRevLett. 105.151101.

[58] H. Schoorlemmer, et al., Energy and Flux Measurements of Ultra-High Energy Cosmic Rays Observed During the First ANITA Flight, Astropart. Phys. 77 (2016) 32-43. arXiv: 1506.05396, doi: 10.1016/j . astropartphys.2016.01.001.

[59] S. W. Barwick, et al., Radio detection of air showers with the ARIANNA experiment on the Ross Ice Shelf, Astropart. Phys. 90 (2017) 50-68. arXiv: 1612 .04473, doi:10.1016/j . astropartphys.2017.02.003.

[60] S. W. Barwick, et al., Observation of classically 'forbidden' electromagnetic wave propagation and implications for neutrino detection, JCAP 1807 (07) (2018) 055. arXiv: 1804.10430, doi : 10 . 1088/1475-7516/2018/07/055.

[61] P. Allison, et al., Measurement of the real dielectric permittivity $\epsilon_{r}$ of glacial ice, Astropart. Phys. 108 (2019) 63-73. arXiv:1712.03301, doi:10.1016/j .astropartphys. 2019. 01.004.

[62] J. Avva, et al., Development Toward a Ground-Based Interferometric Phased Array for Radio Detection of High Energy Neutrinos, Nucl. Instrum. Meth. A869 (2017) 46-55. arXiv: 1605.03525, doi:10.1016/j.nima.2017.07.009.

[63] C. Deaconu, et al., Measurements and Modeling of Near-Surface Radio Propagation in Glacial Ice and Implications for Neutrino Experiments, Phys. Rev. D98 (4) (2018) 043010. arXiv: 1805.12576, doi:10.1103/PhysRevD.98.043010.

[64] D. Smith, et al., Hardware Development for the Radio Neutrino Observatory in Greenland (RNO-G), PoS ICRC2021 (2021) 1058. doi : 10.22323/1.395.1058.

[65] P. Allison, et al., Design and Performance of an Interferometric Trigger Array for Radio Detection of High-Energy Neutrinos, Nucl. Instrum. Meth. A930 (2019) 112-125. arXiv: 1809.04573, doi:10.1016/j.nima.2019.01.067. 
[66] C. Glaser, S. W. Barwick, An improved trigger for Askaryan radio detectors, JINST 16 (05) (2021) T05001. arXiv:2011.12997, doi:10.1088/1748-0221/16/05/T05001.

[67] J. Roberts, et al., LAB4D: A low power, multi-GSa/s, transient digitizer with sampling timebase trimming capabilities, Nucl. Instrum. Meth. A925 (2019) 92-100. arXiv: 1803. 04600, doi:10.1016/j.nima.2019.01.091.

[68] P. Schellart, et al., Detecting cosmic rays with the LOFAR radio telescope, Astron. Astrophys. 560 (2013) A98. arXiv: 1311.1399, doi : 10.1051/0004-6361/201322683.

[69] A. Aab, et al., Probing the radio emission from air showers with polarization measurements, Phys. Rev. D 89 (5) (2014) 052002. arXiv:1402.3677, doi:10.1103/PhysRevD.89. 052002.

[70] D. García-Fernández, C. Glaser, A. Nelles, The signatures of secondary leptons in radioneutrino detectors in ice (3 2020). arXiv:2003.13442.

[71] K. D. de Vries, et al., The cosmic-ray air-shower signal in Askaryan radio detectors, Astropart. Phys. 74 (2016) 96-104. arXiv: 1503.02808, doi:10.1016/j .astropartphys.2015. 10.003.

[72] J. A. Johnson, et al., A new $122 \mathrm{~mm}$ electromechanical drill for deep ice-sheet coring (disc): 5. experience during greenland field testing, Annals of Glaciology 47 (2007) 54-60.

[73] J. Rix, et al., Development of the British Antarctic Survey Rapid Access Isotope Drill, Journal of Glaciology 65 (250) (2019) 288-298. 


\section{Full Authors List: RNO-G Collaboration}

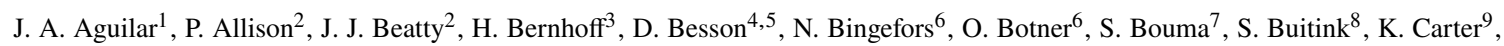
M. Cataldo ${ }^{7}$, B. A. Clark ${ }^{10}$, Z. Curtis-Ginsberg ${ }^{11}$, A. Connolly ${ }^{2}$, P. Dasgupta ${ }^{1}$, S. de Kocker ${ }^{12}$, K. D. de Vries ${ }^{12}$, C. Deaconu ${ }^{11}$, M. A. DuVernois ${ }^{13}$, C. Glaser ${ }^{6}$, A. Hallgren ${ }^{6}$, S. Hallmann ${ }^{14}$, J. C. Hanson ${ }^{15}$, B. Hendricks ${ }^{16}$, B. Hokanson-Fasig ${ }^{13}$, C. Hornhuber ${ }^{4}$, K. Hughes ${ }^{11}$, A. Karle ${ }^{13}$, J. L. Kelley ${ }^{13}$, S. R. Klein ${ }^{17}$, R. Krebs ${ }^{16}$, R. Lahmann ${ }^{7}$, U. Latif ${ }^{12}$, M. Magnuson ${ }^{4}$, T. Meures ${ }^{13}$, Z. S. Meyers ${ }^{14,7}$, K. Mulrey ${ }^{8}$, A. Nelles ${ }^{14,7}$, A. Novikov ${ }^{4}$, E. Oberla ${ }^{11}$, B. Oeyen ${ }^{18}$, H. Pandya ${ }^{8}$, I. Plaisier ${ }^{7,14}$, L. Pyras ${ }^{14,7}$, D. Ryckbosch ${ }^{18}$, O. Scholten ${ }^{12,19}$, D. Seckel ${ }^{20}$, D. Smith ${ }^{11}$, D. Southall ${ }^{11}$, J. Torres ${ }^{2}$, S. Toscano ${ }^{1}$, D. Tosi ${ }^{13}$, D. J. Van Den Broeck ${ }^{12,8}$, N. van Eijndhoven ${ }^{12}$, A. G. Vieregg ${ }^{12}$, C. Welling ${ }^{7,14}$, S. Wissel ${ }^{16,9}$, R. Young ${ }^{4}$, and A. Zink ${ }^{7}$

${ }^{1}$ Université Libre de Bruxelles, Science Faculty CP230, B-1050 Brussels, Belgium

${ }^{2}$ Dept. of Physics, Center for Cosmology and AstroParticle Physics, Ohio State University, Columbus, OH 43210, USA

${ }^{3}$ Uppsala University, Dept. of Engineering Sciences, Division of Electricity, Uppsala, SE-752 37, Sweden

${ }^{4}$ University of Kansas, Dept. of Physics and Astronomy, Lawrence, KS 66045, USA

${ }^{5}$ National Nuclear Research University MEPhI, Kashirskoe Shosse 31, 115409, Moscow, Russia

${ }^{6}$ Uppsala University, Dept. of Physics and Astronomy, Uppsala, SE-752 37, Sweden

${ }^{7}$ Erlangen Center for Astroparticle Physics (ECAP), Friedrich-Alexander-University Erlangen-Nuremberg, 91058 Erlangen, Germany

${ }^{8}$ Vrije Universiteit Brussel, Astrophysical Institute, Pleinlaan 2, 1050 Brussels, Belgium

${ }^{9}$ Physics Dept. California Polytechnic State University, San Luis Obispo CA 93407, USA

${ }^{10}$ Dept. of Physics and Astronomy, Michigan State University, East Lansing MI 48824, USA

${ }^{11}$ Dept. of Physics, Enrico Fermi Inst., Kavli Inst. for Cosmological Physics, University of Chicago, Chicago, IL 60637, USA

${ }^{12}$ Vrije Universiteit Brussel, Dienst ELEM, B-1050 Brussels, Belgium

${ }^{13}$ Wisconsin IceCube Particle Astrophysics Center (WIPAC) and Dept. of Physics, University of Wisconsin-Madison, Madison, WI 53703, USA

${ }^{14}$ DESY, Platanenallee 6, 15738 Zeuthen, Germany

${ }^{15}$ Whittier College, Whittier, CA 90602, USA

${ }^{16}$ Dept. of Physics, Dept. of Astronomy \& Astrophysics, Penn State University, University Park, PA 16801, USA

${ }^{17}$ Lawrence Berkeley National Laboratory, Berkeley, CA 94720, USA

${ }^{18}$ Ghent University, Dept. of Physics and Astronomy, B-9000 Gent, Belgium

${ }^{19}$ Kapteijn Institute, University of Groningen, Groningen, The Netherlands

${ }^{20}$ Dept. of Physics and Astronomy, University of Delaware, Newark, DE 19716, USA 